\title{
Are pigs a suitable model species for osteoporosis research?
}

\author{
Sipos $\mathrm{W}^{*}$ \\ Clinical Department for Farm Animals, University of Veterinary Medicine Vienna, Austria
}

Osteoporosis is a systemic disease, mainly affecting the bone apparatus of postmenopausal women. The aetiology of postmenopausal osteoporosis is complex, but mainly relies on hypoestrogenism in combination with secondary hyperparathyroidism, at least in Western countries, and is associated with an aberrant immune status [1]. As a result of seeking for the main promotors of osteoporosis at the molecular level, a cytokine termed receptor activator of nuclear factor $\kappa \mathrm{B}$ ligand (RANKL), which is a member of the tumor necrosis factor superfamily and a master regulator of osteoclastogenesis, has been identified some 20 years ago and thus initiated osteoimmunology-based research for novel treatment options for osteoporosis and associated pathologies $[2,3]$.

As pigs are increasingly becoming utilized in biomedical research it was logical to test them for their suitability as model species for postmenopausal osteoporosis research. Their suitability for that purpose seemed to be underlined by several physiological facts, such as evidence for trabecular and cortical (Haversian) bone remodeling and a regular, spontaneous estric cycle. Additionally, the existence of the aforementioned cytokine RANKL, which is part of the RANK-RANKLOPG system and thus essential for osteoclastogenesis in rodents and humans, could be proven to be existent also in pigs [4]. There are several methods to mimic postmenopausal osteoporosis in large animal models, such as ovariectomy (OVX), nutritive calcium restriction, long-term glucocorticoid (GC) application, and combinations. For pigs, results using such methods were heterogeneous (reviewed in detail by [5]). The development of osteoporosis is judged based on (I) morphological data as obtained by microstructural and histological analyses, (II) chemical bone metabolism marker titers, which should clearly point towards increased bone resorption and decreased bone formation, (III) mechanical testing of bone strength, and (IV) should ideally include osteoimmunological analyses. All studies using OVX and/or calcium restriction performed to date mainly used adolescent minipigs and could show only some minor site-specific changes in bone mineral density or trabecular architecture, sometimes in combination with alterations in bone metabolism marker titers, or only changes in bone markers without a morphological substrate. Primary osteoporosis in humans is also associated with spontaneous bone fractures, which as an additional major draw-back cannot be shown in any large animal model. As previous studies were quite inhomogeneous concerning ages of pigs included and the used protocols to induce osteoporosis, our aim was to test, whether a combination of OVX and moderate nutritive calcium restriction, thus mimicking the situation in postmenopausal women in many Western countries, was able to induce an osteoporotic state in adult conventional sows as opposite to the mainly used adolescent Göttingen minipigs, as these are chondrodystrophic dwarfs on the one hand and do not represent post-menopausal women by age on the other hand. As peak bone mass in pigs is obtained with an age of 2-3 years, we included multiparous sows aged 33 months in the mean in our study. Additionally we tested for alterations in the immune system by means of flow cytometric analyses of PBMC populations and systemic cytokine profiles due to the afore-mentioned importance of the immune system for bone health and disease, which had not been done before in pigs. Unsatisfactorily, we could not induce any osteoporotic phenotype or changes in cellular immunity in these animals [6]. On the other hand, GC treatment in adult minipigs induces significant changes in bone architecture and strength, thus partially resembling osteoporosis in postmenopausal women [7]. These authors could also clearly demonstrate the protective effect of ibandronate as reference treatment option in that model [8]. A draw-back for the GC-induced osteoporosis model is, however, the systemic influence of GCs on the immune system, which additionally hampers osteoimmunological investigations and therefore does not allow a holistic view on the antiosteoporotic actions of tested novel substances.

When examinig the effects of osteoprotegerin (OPG), which is the natural antagonist to RANKL and thus an inhibitor of osteoclastogenesis, in growing pigs, we could clearly demonstrate the hypothesized effects, i.e. a decrease of bone resorption (and formation due to coupling effects) markers and serum calcium levels. Additionally, $\mu \mathrm{CT}$ analyses demonstrated an improvement of specific architectural indices and bone mineralization at selected bone sites [9]. Research with OPG led to the development of denosumab, which is a fully humanized monoclonal antibody against RANKL and licensed for the treatment of osteoporosis with an elevated risk for fractures in post-menopausal women as well as in men. Meanwhile, research for novel therapeutic options for the treatment of osteoporosis focuses on osteoblastogenetic pathways [2].

When summarizing these observations we can see that adult commercial sows seemingly are no suitable model species for postmenopausal osteoporosis research. The reasons for that could be a generally higher bone mineral density in pigs when compared to humans and also an (at least partial) extragonadal estrogen synthesis, which plays OVX off against the desired hypoestrogenism [6]. On the other hand, bone architecture and metabolism clearly react to osteoimmunological measures as expected at least in adolescent commercial pigs, as shown for OPG [9]. Our group used this observation to test pigs aged 2-4 months as a model for a type of secondary osteoporosis, namely alcohol-induced osteoporosis [10]. In this model we observed the effects of voluntary alcohol binge-drinking on bone. Serum calcium and phosphate levels were decreased in the

${ }^{\star}$ Correspondence to: Wolfgang Sipos, DVM, PhD, Dipl.ECPHM, Clinical Department for Farm Animals, University of Veterinary Medicine Vienna, Veterinarplatz 1, 1210 Vienna, Austria,E-mail: wolfgang.sipos@vetmeduni.ac.at

Received: March 01, 2019; Accepted: March 18, 2019; Published: March 21, 2019 
alcohol group. Bone effects were site-specific. As group sizes in this pilot study were quite small, a study with a higher number of animals per group should be added to be able to draw clear conclusions on bone effects of intense alcohol consumption in that model.

So, when considering pigs as models for osteoporosis research, one has to keep in mind what kind of osteoporosis should be addressed. Under the assumption that GC-induced osteoporosis is not reflecting the situation in post-menopausal women accurately, also due to the immunosuppressive effects of GCs, pigs seem not to be fitting as ideal models for primary osteoporosis, but may be of value when examining (I) GC-induced osteoporosis as pathologic entity by itself, (II) other secondary osteoporotic syndromes such as alcohol-induced osteoporosis, or (III) testing principal effects of pharmaceuticals as well as biologicals on bone biology, which can allow some conclusions on their suitability for osteoporosis treatment. Due to the higher plasticity of bone in prepubescent pigs, outcomes of studies regarding topics related to afore-mentioned points II and III should be more precise when using animals of that age group. Concerning pharmacological studies, many rodent models for sure are advantageous over pigs as they are much cheaper and exhibit similar reactions to boneactive pharmaceuticals as humans. However, in accordance to FDA regulations [11] the use of a larger model species is demanded, mainly due to biomechanical reasons, besides rodents in preclinical testing of antiosteoporotic substances, and thus growing pigs are a useful option for that issue.

\section{References}

1. Sipos W, Pietschmann P, Rauner M, Kerschan-Schindl K, Patsch J (2009) Pathophysiology of osteoporosis. Wien Med Wochenschr 159: 230-234. [Crossref]
2. Rauner M, Sipos W, Thiele S, Pietschmann P (2013) Advances in osteoimmunology: pathophysiologic concepts and treatment opportunities. Int Arch Allergy Immunol 160: 114-125. [Crossref]

3. Sipos W, Pietschmann P, Rauner M (2008) Strategies for novel therapeutic approaches targeting cytokines and signaling pathways of osteoclasto- and osteoblastogenesis in the fight against immune-mediated bone and joint diseases. Curr Med Chem 15: 127136. [Crossref]

4. Sipos W, Duvigneau JC, Schmoll F, Exel B, Hofbauer G, Baravalle G, et al. (2005) Characterization of the cytokine pattern of porcine bone marrow-derived cells treated with 1 $\alpha, 25(\mathrm{OH})_{2} \mathrm{D}_{3}$. J Vet Med A Physiol Pathol Clin Med 52: 382-387.

5. Sipos W (2016) Antibodies for the treatment of bone diseases: Preclinical Data. In: Pietschmann, P (Ed.) Principles of Osteoimmunology. Springer Int., $2^{\text {nd }}$ edn, 217-237.

6. Sipos W, Kralicek E, Rauner M, Duvigneau CJ, Worliczek HL, Schamall D, et al. (2011) Bone and cellular immune system of multiparous sows are insensitive to ovariectomy and nutritive calcium shortage. Horm Metab Res 43: 404-409. [Crossref]

7. Scholz-Ahrens KE, Delling G, Stampa B, Helfenstein A, Hahne HJ, Açil Y, et al. (2007) Glucocorticosteroid-induced osteoporosis in adult primiparous Gottingen miniature pigs: effects on bone mineral and mineral metabolism. Am J Physiol Endocrinol Metab 293: 385-395. [Crossref]

8. Gluer CC, Scholz-Ahrens KE, Helfenstein A, Delling G, Timm W, Acil Y, et al. (2007) Ibandronate treatment reverses glucocorticoid-induced loss of bone mineral density and strength in minipigs. Bone 40: 645-655.

9. Sipos W, Zysset P, Kostenuik P, Mayrhofer E, Bogdan C, Rauner M, et al. (2011) OPG-Fc treatment in growing pigs leads to rapid reductions in bone resorption markers, serum calcium, and bone formation markers. Horm Metab Res 43: 944-949. [Crossref]

10. Foger-Samwald U, Knecht C, Stimpfl T, Szekeres T, Kerschan-Schindl K, Mikosch P, et al. (2018) Bone Effects of Binge Alcohol Drinking Using Prepubescent Pigs as a Model. Alcohol Clin Exp Res 42: 2123-2135.

11. Guidelines for preclinical and clinical evaluation of agents used for the prevention or treatment of postmenopausal osteoporosis. Division of Metabolism and Endocrine Drug Products, Food and Drug Administration. 1994. 